\title{
荒漠草地植物多样性对草食动物采食的响应机制
}

\author{
刘文亭 1 卫智军 ${ }^{1 *}$ 吕世杰 ${ }^{2}$ 孙世贤 $^{3}$ 贾利娟 ${ }^{4}$ 张爽 $^{1}$ 王天乐 $^{1}$ 代景忠 $^{1}$ \\ 卢志宏 ${ }^{1}$
}

${ }^{1}$ 内蒙古农业大学生态环境学院, 呼和浩特 010019; ${ }^{2}$ 内蒙古农业大学理学院, 呼和浩特 010018; ${ }^{3}$ 中国农业科学院草原研究所, 呼和浩特 $010010 ;{ }^{4}$ 苏 尼特右旗草原工作站, 内蒙古锡林郭勒 011200

摘 要 草食动物采食对草地植物多样性和生态系统功能的影响机制是放牧生态学研究的核心问题。该研究以内蒙古锡林郭 勒盟苏尼特右旗荒漠草原的长期放牧控制实验为平台, 从既有草地植物多样性和动物偏食性两个层面系统地研究了荒漠草 地植物多样性对草食动物采食的响应机制。结果显示: 1)荒漠草地植物对草食动物采食呈现4种响应模式: 放牧“隐没种”、放 牧“敏感种”、放牧“无感种”、“绝对优势种”; 2)在群落尺度上, 物种多样性指数随放牧强度增加而减少, 与不放牧小区相比, 重 度放牧(HG)与适度放牧(MG)小区植物多样性均下降, 且这一规律同样适用于功能群多样性, 灌木半灌木这一功能群内物种 多样性对放牧干扰较敏感; 3)在草地既有植物的基础上, 以不放牧小区为参考系, 草食动物对植物功能群偏食性的排序为: 一二年生草本 $(\mathrm{AB})>$ 多年生杂类草 $(\mathrm{PF})>$ 灌木半灌木 $(\mathrm{SS})>$ 多年生禾草(PG), 且偏食性物种主要分布于 $\mathrm{AB}$ 和PF中; 4)植物多 样性与动物偏食性基本呈显著负相关关系 $(p<0.05)$ 。

关键词 植物多样性; 偏食性; 草食动物; 荒漠草地; 组织尺度

引用格式: 刘文亭, 卫智军, 吕世杰, 孙世贤, 贾利娟, 张爽, 王天乐, 代景忠, 卢志宏 (2016). 荒漠草地植物多样性对草食动物采食的响应机制. 植 物生态学报, 40, 564-573. doi: 10.17521/cjpe.2015.0421

\section{Response mechanism of plant diversity to herbivore foraging in desert grassland}

LIU Wen-Ting ${ }^{1}$, WEI Zhi-Jun ${ }^{1 *}$, LÜ Shi-Jie ${ }^{2}$, SUN Shi-Xian ${ }^{3}$, JIA Li-Juan ${ }^{4}$, ZHANG Shuang ${ }^{1}$, WANG Tian-Le ${ }^{1}$, DAI Jing-Zhong ${ }^{1}$, and LU Zhi-Hong ${ }^{1}$

${ }^{1}$ College of Ecology and Environmental Science, Inner Mongolia Agricultural University, Hohhot 010019, China; ${ }^{2}$ College of Science, Inner Mongolia Agricultural University, Hohhot 010018, China; ${ }^{3}$ Institute of Grassland Research, Chinese Academy of Agriculture Sciences, Hohhot 010010, China; and ${ }^{4}$ Sonid Right Banner Station of Grassland Management, Xilingol, Nei Mongol 011200, China

\section{Abstract}

Aims The mechanism of herbivore foraging effect on plant diversity and ecosystem functions of grasslands is the core issue of grazing ecology research. This study is aimed to examine 1) how species respond to different grazing intensity in grassland, and how plant diversity responds to different grazing intensity within different organization scales and whether there are cascade effects across multiple organization scales? 2) How herbivore foraging affects plant diversity.

Methods Based on a six-year grazing experiment in Sonid Right Banner, Xilingol, the study attempted to explain the relationships between plant diversity and herbivore foraging from two perspectives, i.e. plant diversity index and herbivore preference index.

Important findings Results showed that there were four modes for desert grassland plant in response to herbivore: grazing-hidden species, grazing-sensitive species, grazing-non inductive species and absolute dominant species. At the community scale, species diversity index decreased with an increased grazing intensity. Compared with no grazing, heavy grazing and moderate grazing treatments decreased community plant diversity, and the same principle is applicable to the functional group diversity. Further analysis found that differences of grazing species diversity within a functional group were mainly caused by shrubs and semi-shrubs. Compared to plots without grazing, the herbivore preference degree on the whole species was lower for moderate grazing plots than heavy grazing ones. The foraging preference order on the plant functional groups was annual and biennial forbs $(\mathrm{AB})$, perennial forbs (PF), shrubs and semi-shrubs (SS) and perennial grasses (PG). The preferred species were mainly distributed in $\mathrm{AB}$ and PF. The diversity index of plant and preference index of herbivore were basically

收稿日期Received: 2015-11-23 接受日期Accepted: 2016-05-12

* 通信作者Author for correspondence (E-mail: nmndwzj@163.com) 
significant and negatively correlated $(p<0.05)$.

Key words plant diversity; preference species; herbivore; desert grassland; organization scale

Citation: Liu WT, Wei ZJ, LÜ SJ, Sun SX, Jia LJ, Zhang S, Wang TL, Dai JZ, Lu ZH (2016). Response mechanism of plant diversity to herbivore foraging in desert grassland. Chinese Journal of Plant Ecology, 40, 564-573. doi: 10.17521/cjpe.2015.0421

植物多样性是草地生态系统功能的核心内容之 一, 保护生物多样性亦为当前世界广泛关注的重要 科学问题(Hector \& Schmid, 1999; Chapin et al., 2000; Storkey et al., 2015; Tilman \& Isbell, 2015)。草 食动物作为草地植物群落组成与多样性的直接管理 者, 其采食行为是影响植物群落种群动态和多样性 变化的最直接因素(Hodgson \& Illius, 1996)。合理的 放牧能够促进草地营养物质的循环、非生物与生物 资源的转化和维持草地的多功能以及系统的可持续 性(Jared et al., 2015)。

在探讨草食动物与植物关系时, 不同学者从单 一的放牧组合、放牧频率、放牧周期等视角 (Rossignol et al., 2006; 许岳飞等, 2012; 孙世贤等, 2013), 到基于降水量(丰水年份、平水年份、欠水年 份)、地形(平地和坡地)与放牧调控相结合的方式, 已系统地分析了植物物种、功能群及群落多样性对 放牧的适应机制(Wan et al., 2011; Bai et al., 2012; 杨婧等, 2014)。尽管这些研究丰富了草地植物多样 性理论, 但仅局限于植物这一层面中。草食动物通 过采食(下行作用)作用于局部植物生存与灭绝来动 态调控植物多样性(Olff \& Ritchie, 1998), 同时也受 植物(上行作用)的作用来维持草地植物多样性与生 态系统过程(Chapin et al., 2000; Gibson, 2009; Jared et al., 2015), 它们之间相互作用、彼此依托共同组 成一个反馈回路(feedback loop)。这就提供了一个假 说, 草食动物对草地植物多样性造成影响, 而既有 现存植物可体现家畜对某些植物的偏好。事实上, 现有放牧研究体系中, 在动物对植物多样性的影响 过程中, 单独抽离出植物多样性对动物的影响仍极 具挑战(王岭, 2010)。动物在非限制性条件下所做的 食物选择、持续单一稳定的食性选择方式, 可能是 导致草地偏食物种局部灭绝的重要原因之一, 尤其 当偏食物种为稀有种或非耐牧种时, 这种灭绝的风 险可能就越大; 同时在衡量现存植物群落的多样性 研究中, 草地本身的优势种和耐牧种的数量特征才 是判断其植物多样性高低的决定性因素(Louault et al., 2005)。
此外, 放牧对植物的影响体现在草地生命系统 的各尺度上(马银山和张世挺, 2009; Wan et al., 2011), 不同尺度的多样性是紧密联系、不可分割的, 某一尺度的多样性是由其下一级生命实体的不同组 合方式形成的(马克平, 1993)。换言之, 植物多样性 受放牧影响而产生的变化, 将经由物种尺度导致功 能群尺度、群落尺度甚至生态系统尺度的级联反应。 此外, 单个尺度仅能反映植被本身综合特征的一个 方面, 并不能系统性地还原问题本质, 充分结合植 物在不同组织尺度的收缩或扩张来进行分析, 将更 有利于理解和简化生态系统过程(刘文亭等, 2015)。

草食动物的采食行为是一个非常复杂的过程, 在采食过程中, 家畜需要在不同植株个体间重复选 择, 选择的结果直接影响其生存质量(Stephens \& Krebs, 1986)。研究认为, 植物种类较多时, 动物拥 有更多的选项来挑剔其喜食的物种或物种组合 (Provenza, 1995), 也可频繁地在不同物种间进行尝 试。那么, 草食动物是否存在固定的采食模式呢? 随着家畜个体数量的增加, 该模式是否一直可行 呢? 本研究依托于内蒙古锡林郭勒盟苏尼特右旗的 短花针茅(Stipa breviflora) 荒漠草原建立的长期放牧 平台作为模式生态系统, 从草地既有现存植物多样 性的状态入手, 来推测草食动物对植物的偏食性, 以期回答以下问题: 草地群落植物物种对不同放牧 强度如何响应? 植物多样性对放牧强度的响应有何 不同? 不同尺度植物多样性对放牧强度的响应又有 何差异? 结合家畜的偏食性, 深度剖析荒漠草地植 物多样性对放牧的响应机制, 以期对制定干旱区荒 漠草地的放牧管理制度提供数据支撑与理论基础。

\section{1 材料和方法}

\section{1 研究区概况}

研究区位于内蒙古锡林郭勒盟苏尼特右旗朱日 和镇 $\left(112.79^{\circ} \mathrm{E}, 42.26^{\circ} \mathrm{N}\right)$ 的牧区。该地区地形较平 坦, 有明显的钙积层, 钻积层大部分分布在地下 10-80 cm。土壤为淡栗钙土, 腐殖质层厚5-10 cm。 2012-2014年平均年降水量 $230.9 \mathrm{~mm}, 60 \%-80 \%$ 的 
降水集中在牧草生长的旺季(7-9月), 蒸发量2 500 $\mathrm{mm}$ 。2012-2014年月平均气温较为接近, 年平均气 温 $5.9{ }^{\circ} \mathrm{C}$, 月平均气温最高 $24.45{ }^{\circ} \mathrm{C}$, 最低气温 $-16.28{ }^{\circ} \mathrm{C}$ 。以短花针茅为建群种, 优势种为无芒隐 子草(Cleistogenes songorica)、碱非(Allium polyrhizum)等, 具体见表1。

\section{2 试验地设置与取样方法}

本研究的草食动物为绵羊品种 “苏尼特羊”。放 牧实验于2010年开始, 每年5月开始放牧, 10月底终 止, 期间采用连续放牧方式, 夜间羊群不归牧, 不 进行补饲。试验平台设有放牧季节调控、放牧强度 调控与放牧强度下季节调控 3 种实验方案(孙世贤, 2013), 每个处理均设有3次重复, 每个实验小区面 积 $2.60 \mathrm{hm}^{2}$ 。放牧区绵羊的健康状况、个体大小、 体重、性别基本一致。

本试验试图从既有不同放牧强度草地植物多样 性现状, 及反推造成这一现状的草食动物的食性选
择的多维度视角来解释植物多样性对放牧的响应机 制，并不涉及放牧年限及降水因素的干扰，故只抽 取全年重度放牧(HG)、全年适度放牧(MG)、全年不 放牧(NG)这3种处理方式，并选用放牧试验第3年、 第4年、第5年, 即2012年(丰水年)、2013年(平水年)、 2014 年 (欠水年) 的植物数据, 表 2 为研究区 2012-2014年月降水量变化。其中适度放牧处理和重 度放牧处理载畜率分别为 0.96 和 1.54 只羊 $\cdot \mathrm{hm}^{-2}$ 。于 2010年放牧实验开始前在每个小区内随机设置 5 个 1 $\mathrm{m} \times 1 \mathrm{~m}$ 固定样方, 用涂有红色油漆的木桩标记好 每个样方的具体位置, 同时在小区内随机调查 5 个 1 $\mathrm{m} \times 1 \mathrm{~m}$ 样方(减少固定样方因羊采食行为随时间累 积的草地异质性所带来的实验误差)。样方调查在荒 漠草地植物生长最高峰期的8月份进行。每个样方的 主要调查指标为物种数、密度 (从生型植物为分株 数, 同时记录株从大小; 非丛生型禾草植物为个体 数)。

表1 研究地存在物种表

Table 1 Existing plants of the study site

\begin{tabular}{|c|c|c|c|}
\hline $\begin{array}{l}\text { 物种 } \\
\text { Species } \\
\end{array}$ & $\begin{array}{l}\text { 植物功能群 } \\
\text { Plant functional groups }\end{array}$ & $\begin{array}{c}\text { 物种代码 } \\
\text { Species code }\end{array}$ & $\begin{array}{c}\text { 植物功能群代码 } \\
\text { Plant functional group code }\end{array}$ \\
\hline 短花针茅 Stipa breviflora & 多年生草本 Perennial grasses & $\mathrm{S} 1$ & PG \\
\hline 无芒隐子草 Cleistogenes songorica & 多年生草本 Perennial grasses & $\mathrm{S} 2$ & PG \\
\hline 寸草 Carex duriuscula & 多年生草本 Perennial grasses & S3 & PG \\
\hline 木地肤 Kochia prostrata & 灌木半灌木 Shrubs and semi-shrubs & S4 & SS \\
\hline 狭叶锦鸡儿 Caragana stenophylla & 灌木半灌木 Shrubs and semi-shrubs & S5 & SS \\
\hline 戈壁天门冬 Asparagus gobicus & 灌木半灌木 Shrubs and semi-shrubs & S6 & SS \\
\hline 茵陈蒿 Artemisia capillaris & 多年生杂类草 Perennial forbs & S7 & $\mathrm{PF}$ \\
\hline 阿尔泰狗娃花 Heteropappus altaicus & 多年生杂类草 Perennial forbs & S8 & $\mathrm{PF}$ \\
\hline 碱非 Allium polyrhizum & 多年生杂类草 Perennial forbs & S9 & $\mathrm{PF}$ \\
\hline 二刺叶兔唇花 Lagochilus diacanthophyllus & 多年生杂类草 Perennial forbs & $\mathrm{S} 10$ & $\mathrm{PF}$ \\
\hline 银灰旋花 Convolvulus ammannii & 多年生杂类草 Perennial forbs & S11 & $\mathrm{PF}$ \\
\hline 二型叶棘豆 Oxytropis diversifolia & 多年生杂类草 Perennial forbs & $\mathrm{S} 12$ & $\mathrm{PF}$ \\
\hline 乳白黄耆 Astragalus galactites & 多年生杂类草 Perennial forbs & $\mathrm{S} 13$ & $\mathrm{PF}$ \\
\hline 拐轴鸦葱 Scorzonera divaricata & 多年生杂类草 Perennial forbs & $\mathrm{S} 14$ & $\mathrm{PF}$ \\
\hline 冷蒿 Artemisia frigida & 多年生杂类草 Perennial forbs & S15 & $\mathrm{PF}$ \\
\hline 蒙古非 Allium mongolicum & 多年生杂类草 Perennial forbs & S16 & $\mathrm{PF}$ \\
\hline 非 Allium tuberosum & 多年生杂类草 Perennial forbs & S17 & $\mathrm{PF}$ \\
\hline 细叶非 Allium tenuissimum & 多年生杂类草 Perennial forbs & S18 & $\mathrm{PF}$ \\
\hline 二裂委陵菜 Potentilla bifurca & 多年生杂类草 Perennial forbs & S19 & $\mathrm{PF}$ \\
\hline 栉叶蒿 Neopallasia pectinata & 一二年生草本 Annual and biennial forbs & S20 & $\mathrm{AB}$ \\
\hline 猪毛菜 Salsola collina & 一二年生草本 Annual and biennial forbs & S21 & $\mathrm{AB}$ \\
\hline 陇牛儿苗 Erodium stephanianum & 一二年生草本 Annual and biennial forbs & S22 & $\mathrm{AB}$ \\
\hline 狗尾草 Setaria viridis & 一二年生草本 Annual and biennial forbs & $\mathrm{S} 23$ & $\mathrm{AB}$ \\
\hline 画眉草 Eragrostis pilosa & 一二年生草本 Annual and biennial forbs & $\mathrm{S} 24$ & $\mathrm{AB}$ \\
\hline
\end{tabular}

www.plant-ecology.com 
表2 研究区2012-2014年月降水量变化(mm)

Table 2 Variations of monthly precipitation of the study site in 2012 to 2014 (mm)

\begin{tabular}{ccccccccccccc}
\hline 年份 & 1月 & 2月 & 3月 & 4月 & 5月 & 6月 & 7月 & 8月 & \multicolumn{2}{c}{ 9月 } & 10月 & 11月 \\
Year & Jan. & Feb. & Mar. & Apr. & May & June & July & Aug. & Sept. & Oct. & Nov. & Dec. \\
\hline 2012 & 0 & 0.1 & 10.2 & 7.5 & 18.7 & 42.7 & 166.6 & 17.3 & 33.2 & 6.6 & 21.1 & 4.7 \\
2013 & 1 & 0.8 & 2.6 & 2.7 & 1.2 & 86.9 & 44.9 & 60.9 & 20.8 & 0.1 & 6.6 & 0.5 \\
2014 & 0 & 7.2 & 0 & 11.6 & 18.9 & 15.1 & 17.7 & 12.7 & 18.8 & 11.6 & 1.1 & 0.3 \\
\hline
\end{tabular}
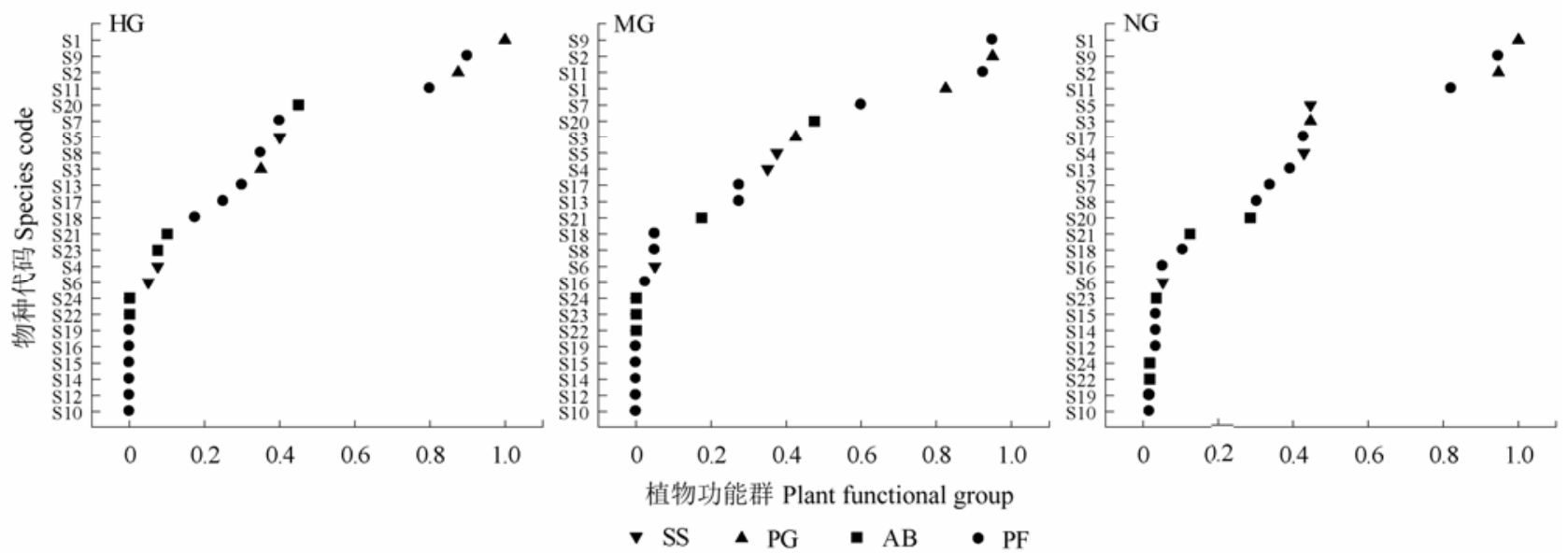

图1＼cjkstart重度放牧(HG)、适度放牧(MG)与不放牧(NG)条件下植物多重响应频数排序。物种及功能群代码同表1。

Fig. 1 Sorting of plant multiple response frequency in heavy grazing (HG), moderate grazing (MG) and no grazing (NG). Species and plant functional group code see Table 1.

\section{3 数据处理与统计}

\subsection{1 物种频数的计算}

本研究采用多重响应分析(multiple response)来 计算草地物种频数(邓荣根, 2012)。多重响应分析本 质属分类数据的再分析, 但由于各选项均是对同一 个问题的回答, 即草地中各物种对草食动物来说是 “采食”或“不采食”，且各物种之间存在一定的相关 性, 因此, 通过定义样方中存在物种的方式来进行 多重响应变量集的交叉表分析是较为合适本研究物 种频数的统计方法。首先对试验处理下的物种进行 $0 、 1$ 赋值来定义变量集, $0 、 1$ 分别代表样方中特定植 物不存在或存在状态; 然后按放牧梯度将变量集分 为3类, 即HG、MG、NG; 最后利用SPSS 20.0 多重 响应分析交叉表分析过程进行分析。

\subsection{2 植物多样性与动物偏食性指数的计算}

植物多样性的测定仅使用丰富度指数与 Shannon-Wiener多样性指数(本文简称多样性指 数)(孙儒泳等, 1993)来表征。依据不同物种的采食情 况, 计算绵羊对不同植物物种的偏食性指数 (preference index, PI)(Wang et al., 2010)。PI指每个物 种的采食情况占所有物种总采食量的百分比, 本研
究以物种的密度来计算, 由于荒漠草地多年生植物 大多为丛生植物, 为了消除多年生植物现存株从大 小 (自身年龄结构或动物采食程度)所带来的差异, 调查时按大丛(基部丛径 $>10 \mathrm{~cm}$ )、中丛(基部丛径 5-10 cm)、小丛(基部丛径 $\leqslant 5 \mathrm{~cm}$ )统计, 然后按 1 中 从 $=3$ 小丛, 1 大从 $=2$ 中从折算为小丛数记为各植物 种群株从密度(吕世杰等, 2014)。

\subsection{3 统计分析}

利用一般线性模型(GLM)分析HG、MG、NG 样地植物多样性的差异性, 并进行Duncan多重比 较。使用Pearson相关分析植物多样性与草食动物的 偏食性相关性, 在0.05和0.01水平进行显著性检验。 以上统计分析均在SPSS 20.0完成。

\section{2 结果}

\section{1 荒漠草地植物多样性对草食动物的响应}

\subsection{1 植物频数的响应特征}

为了了解群落中植物对草食动物的响应, 以草 地既有的24种植物现状为基础, 对不同放牧强度处 理的物种多重响应频数进行了分析(图1), 结果显示 出4种不同的响应类型：1)放牧“隐没种”, 无论是重 
度放牧或适度放牧，二刺叶兔唇花、二型叶棘豆、 拐轴鸦葱、冷蒿、二裂委陵菜、陇牛儿苗、画眉草 均未出现，且主要集中于多年生杂类草和一二年生 草本两个功能群中; 2)放牧“敏感种”, 包括木地肤、 阿尔泰狗娃花、蒙古非、韭、栉叶蒿、狗尾草, 这 类响应频数随放牧强度增加而增加(或减少); 3)放 牧“无感种”, 这类植物频数不随外界干扰发生明显 变动, 如寸草、狭叶锦鸡儿、戈壁天门冬、猪毛菜; 4)“绝对优势种”, 在荒漠草地占有绝对主导地位, 且不轻易随草食动物干扰出现变化, 频数稳定在 0.8 以上, 包含短花针茅、无芒隐子草、碱非、银灰旋 花。

\subsection{2 草地现存植物多样性}

在群落尺度上, 物种丰富度指数无显著性差异

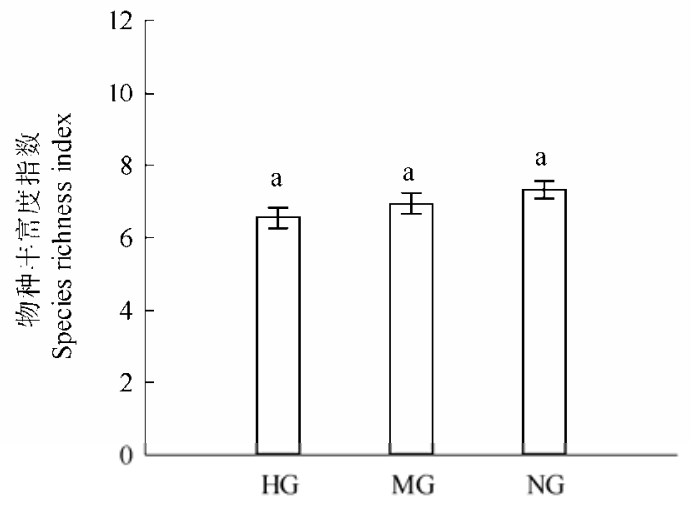

放牧强度 Grazing intensity
(图2), 重度放牧下物种多样性指数显著降低 $(p<$ $0.05)$; 较不放牧处理, 重度放牧与适度放牧处理植 物多样性指数分别下降 $10.92 \% 、 4.81 \%$ 。功能群丰 富度指数方面(图3), 适度放牧功能群丰富度指数大 于不放牧小区，显著大于重度放牧小区(高4.80\%), 且功能群多样性指数呈现同样的趋势。

进一步分析(表3), 本研究将尺度缩小并聚焦于 每个功能群内物种发现, 仅灌木半灌木内物种丰富 度指数产生变化, 不放牧草地和适度放牧草地显著 大于重度放牧 $(p<0.05)$; 物种多样性指数方面, 重 度放牧草地灌木半灌木和多年生杂类草物种多样性 指数较不放牧小区分别显著下降了 $70.67 \% 、 22.97 \%$, 说明灌木半灌木这一功能群内物种多样性对放牧干 扰较敏感。

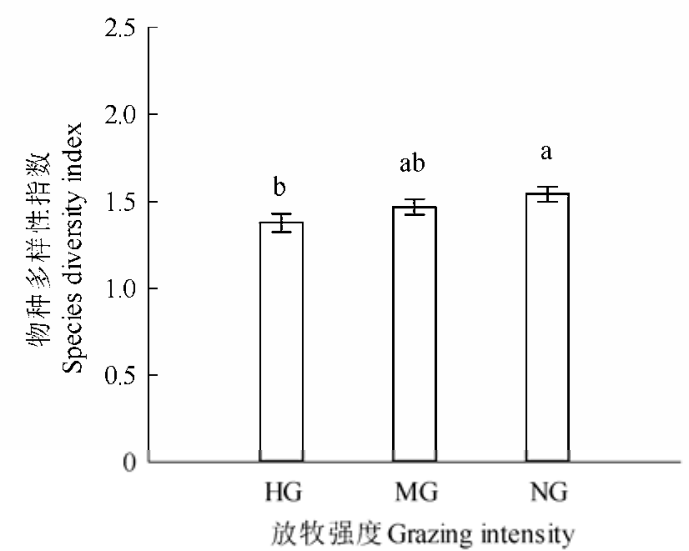

图2 重度放牧( $\mathrm{HG}$ )、适度放牧( $\mathrm{MG}$ )与不放牧(NG)条件下群落内物种多样性(平均值土标准误差)。不同小写字母表示差异显著 $(p<0.05)$ 。

Fig. 2 Species diversity under plant community in heavy grazing (HG), moderate grazing (MG) and no grazing (NG) (mean \pm SE). Different small letters indicate significant differences $(p<0.05)$.

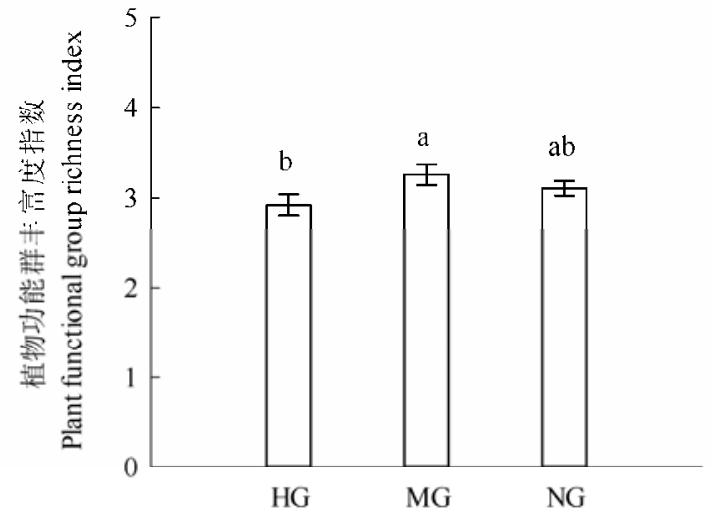

放牧强度 Grazing intensity

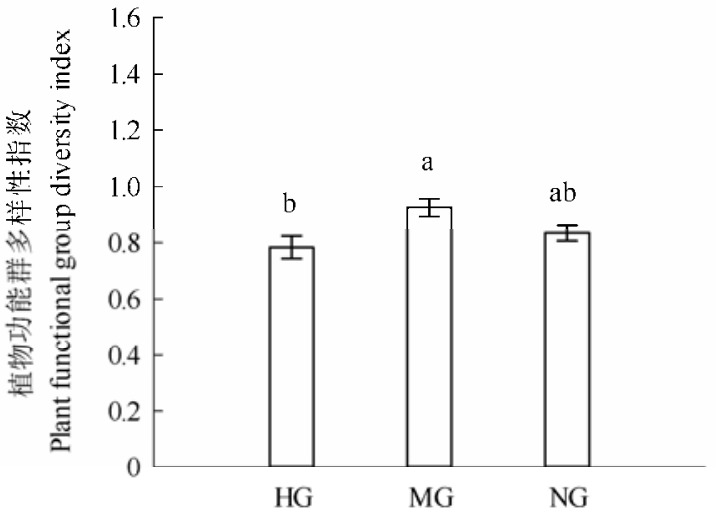

放牧强度 Grazing intensity

图3 重度放牧(HG)、适度放牧(MG)与不放牧(NG)条件下群落内功能群多样性(平均值土标准误差)。不同小写字母表示存在显 著性差异 $(p<0.05)$ 。

Fig. 3 Plant functional group diversity under plant community in heavy grazing (HG), moderate grazing (MG) and no grazing (NG) (mean \pm SE). Different small letters indicate significant differences $(p<0.05)$. 


\section{2 荒漠草地草食动物偏食性变化}

在草地既有植物的基础上，以不放牧小区为参 考系, 从群落与功能群尺度分析了草食动物对各物 种的喜食程度(图4)。草食动物偏食性功能群的排序 为 $\mathrm{AB}>\mathrm{PF}>\mathrm{SS}>\mathrm{PG}$, 且偏食性物种主要分布于 $\mathrm{AB}$ 和PF中, 分别是戈壁天门冬、银灰旋花、二型叶 棘豆、乳白黄耆、拐轴鸦葱、冷蒿、蒙古韭、韭、 细叶韭、二裂委陵菜、陇牛儿苗、狗尾草、画眉草 $(P I>0.60)$ 。进一步分析, 部分植物(短花针茅、寸草、 木地肤、狭叶锦鸡儿)随放牧强度的增加PI减小, 有 的则相反, 如无芒隐子草、阿尔泰狗娃花、栉叶蒿 等, 这说明草食动物的食性选择并非一成不变。

\section{3 荒漠草地植物多样性与动物偏食性的关系}

本研究对荒漠草地植物群落内物种与功能群 多样性与对应的草食动物偏食性进行了 Pearson相 关性分析, 从图5得出, 植物多样性与动物偏食性基
本呈负相关，且负相关对数是正相关对数的6.99倍。 其中在群落内物种尺度上, 负相关对数比正相关对 数多 $866.67 \%$, 群落内物种多样性与偏食性功能群 (多年生禾草、灌木半灌木)显著负相关，与蒙古非显 著正相关; 在群落内功能群尺度方面, 植物多样性 与 4 个功能群的偏食性均显著负相关, 且与短花针 茅、无芒隐子草、狭叶锦鸡儿、茵陈蒿、栉叶蒿、 猪毛菜显著负相关。

\section{3 讨论}

\section{1 荒漠草地物种组成及功能群的频数响应}

荒漠草地不同放牧强度处理植物共 10 科 24 种, 其中禾本科、百合科、菊科植物种类最多(图1)。多 年生丛生禾草的短花针茅、无芒隐子草和百合科的 碱非虽多是矮小的植物, 但对环境有极强的适应力, 共同构成了荒漠草原独特的以强早生植物占绝对优

表3 荒漠草地功能群内物种多样性(平均值土标准误差)

Table 3 Species diversity within plant functional group in desert grassland (mean $\pm S E$ )

\begin{tabular}{|c|c|c|c|c|c|c|c|c|}
\hline \multirow[t]{2}{*}{$\begin{array}{l}\text { 放牧强度 } \\
\text { Grazing intensity }\end{array}$} & \multicolumn{4}{|c|}{ 功能群内物种丰富度指数 } & \multicolumn{4}{|c|}{$\begin{array}{c}\text { 功能群内物种多样性指数 } \\
\text { Species diversity within plant functional groups index }\end{array}$} \\
\hline & $\begin{array}{c}\text { 多年生禾草 } \\
\text { Perennial } \\
\text { grasses }\end{array}$ & $\begin{array}{c}\text { 灌木半灌木 } \\
\text { Shrubs and } \\
\text { semi-shrubs }\end{array}$ & $\begin{array}{l}\text { 多年生杂类草 } \\
\text { Perennial forbs }\end{array}$ & $\begin{array}{c}\text { 二二年生草本 } \\
\text { Annual and } \\
\text { biennial forbs }\end{array}$ & $\begin{array}{c}\text { 多年生禾草 } \\
\text { Perennial } \\
\text { grasses }\end{array}$ & $\begin{array}{c}\text { 灌木半灌木 } \\
\text { Shrubs and } \\
\text { semi-shrubs }\end{array}$ & $\begin{array}{l}\text { 多年生杂类草 } \\
\text { Perennial forbs }\end{array}$ & $\begin{array}{c}\text { 二二年生草本 } \\
\text { Annual and } \\
\text { biennial forbs }\end{array}$ \\
\hline 重度放牧 & $2.26 \pm 0.09^{\mathrm{a}}$ & $0.79 \pm 0.08^{b}$ & $3.23 \pm 0.14^{\mathrm{a}}$ & $0.67 \pm 0.12^{\mathrm{a}}$ & $0.63 \pm 0.04^{\mathrm{a}}$ & $0.04 \pm 0.03^{b}$ & $0.72 \pm 0.04^{\mathrm{b}}$ & $0.15 \pm 0.05^{\mathrm{a}}$ \\
\hline $\begin{array}{l}\text { Heavy grazing } \\
\text { 适度放牧 } \\
\text { Moderate grazing }\end{array}$ & $2.23 \pm 0.1^{\mathrm{a}}$ & $0.53 \pm 0.09^{b}$ & $3.18 \pm 0.25^{\mathrm{a}}$ & $0.63 \pm 0.13^{\mathrm{a}}$ & $0.67 \pm 0.05^{\mathrm{a}}$ & $0.03 \pm 0.03^{\mathrm{ab}}$ & $0.69 \pm 0.07^{\mathrm{b}}$ & $0.13 \pm 0.05^{\mathrm{a}}$ \\
\hline $\begin{array}{l}\text { 不放牧 } \\
\text { No grazing }\end{array}$ & $2.39 \pm 0.07^{\mathrm{a}}$ & $0.93 \pm 0.09^{\mathrm{a}}$ & $3.54 \pm 0.18^{\mathrm{a}}$ & $0.48 \pm 0.09^{\mathrm{a}}$ & $0.68 \pm 0.04^{a}$ & $0.13 \pm 0.04^{\mathrm{a}}$ & $0.93 \pm 0.05^{\mathrm{a}}$ & $0.13 \pm 0.05^{\mathrm{a}}$ \\
\hline
\end{tabular}

同列不同小写字母表示差异显著 $(p<0.05)$ 。

Different small letters in each column indicate significant differences $(p<0.05)$
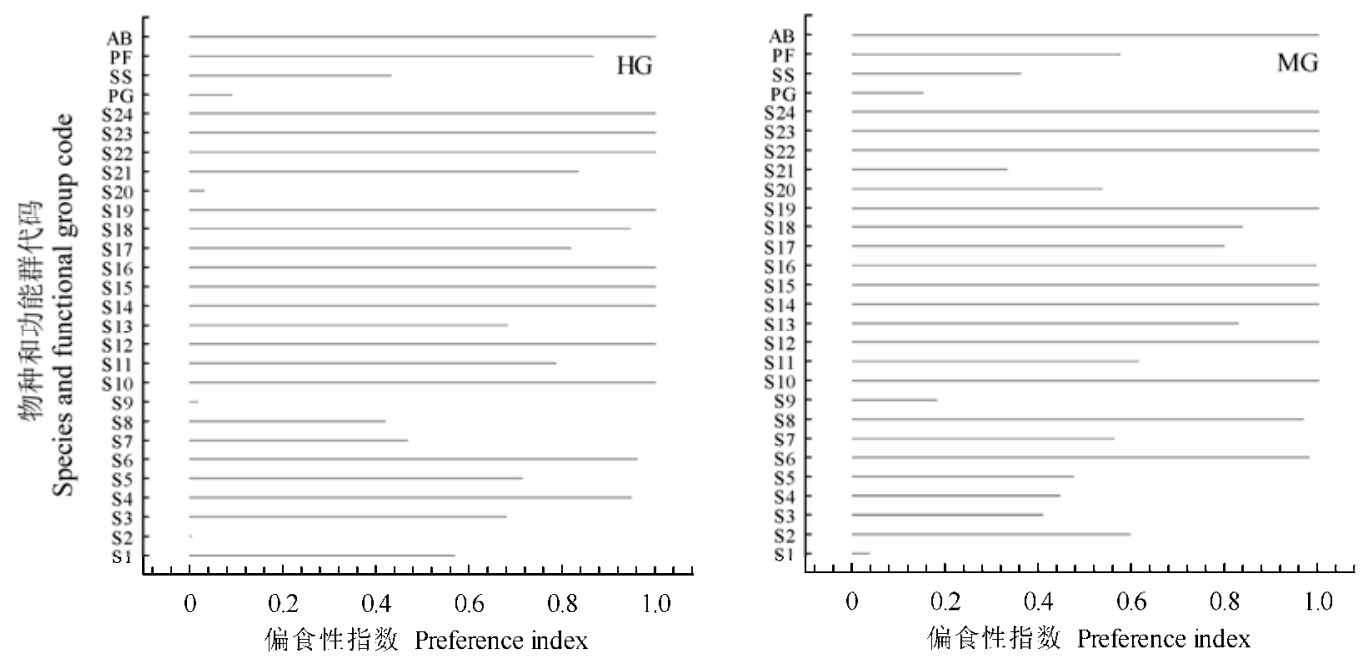

图4 重度(HG)和中度(MG)放牧下荒漠草地草食动物偏食性指数 $(P I)$ 。物种和功能群代码同表 1 。

Fig. 4 Preference index $(P I)$ of herbivore in desert grassland in heavy grazing (HG) and moderate grazing (MG). Species and functional group code see Table 1 . 

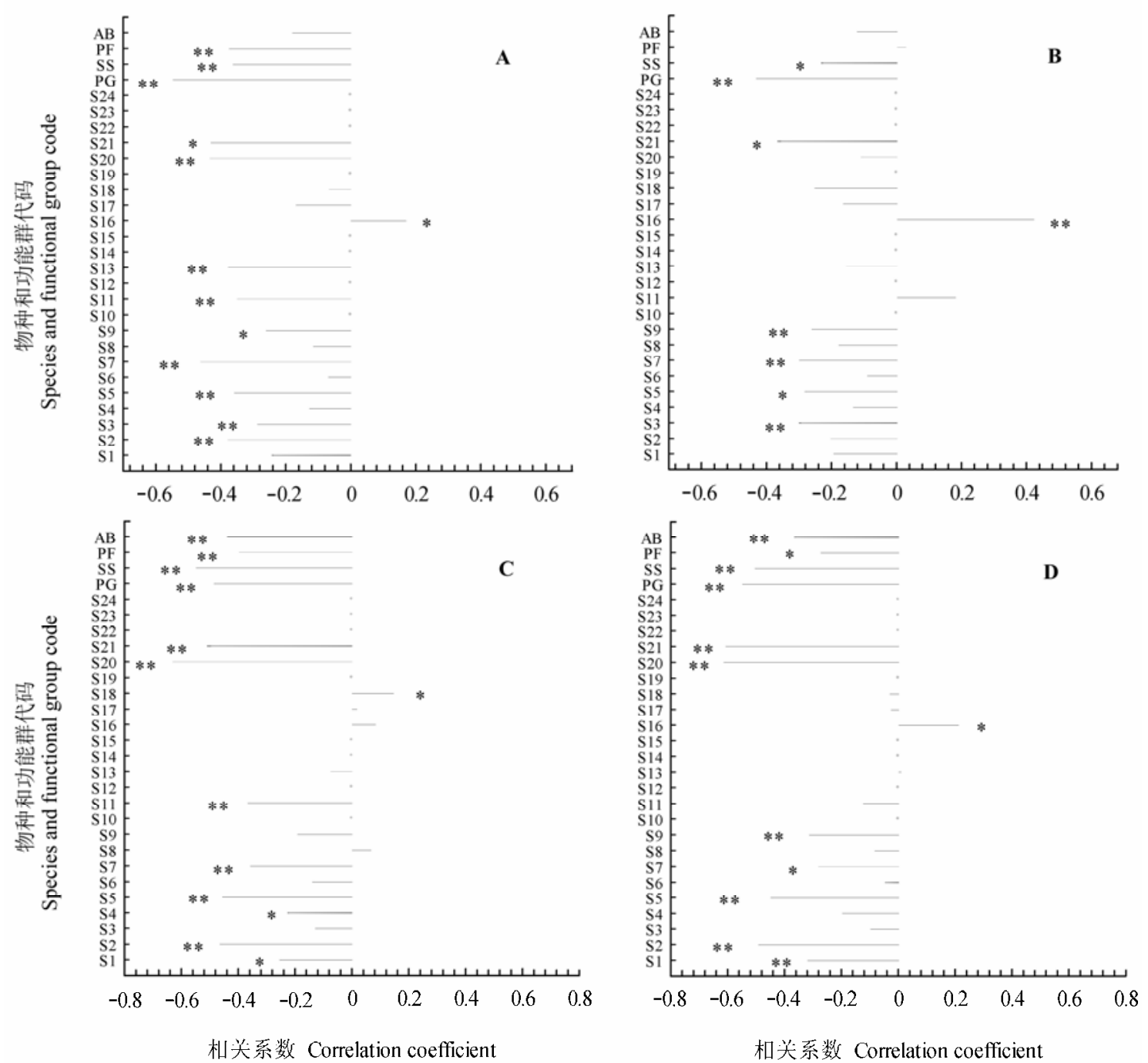

图5 荒漠草地植物多样性与草食动物偏食性的Pearson相关关系。A, 群落内物种丰富度指数与偏食性的Pearson相关关系。 $\mathbf{B}$, 群落内物种多样性指数与偏食性的Pearson相关关系。C, 群落内功能群丰富度指数与偏食性的Pearson相关关系。D, 群落 内功能群多样性指数与偏食性的Pearson相关关系。物种及功能群代码同表 1 。,$p<0.05 ; * * ; p<0.01$ 。

Fig. 5 Pearson relationships between plant diversity and herbivore preference in desert grassland. A, Richness index based on plant species. B, Diversity index based on plant species. C, Richness index based on plant functional groups. D, Diversity index based on plant functional groups. Species code and plant functional groups code see Table 1 . ${ }^{*}, p<0.05 ;{ }^{* *}, p<0.01$.

势的短花针茅+无芒隐子草+碱非群落(卫智军等, 2013; 吕世杰等, 2014)。然而, 动物长期的采食干扰 诱使荒漠草地优势种或优势功能群组成与结构发生 演变, 导致部分“机会主义”物种的绝对或相对丰度 增加(Bai et al., 2012), 如银灰旋花。本研究发现, 某 些机会主义物种因其自身因素(低适口性、低营养含 量; Wang et al., 2010)、环境因素(地表裸露、土壤沙 化、保水能力差; Chen et al., 2013)与动物采食强度 (孙世贤等, 2013)等因素的变化, 使得其响应频数变 化趋势较大, 如蒙古非、韭随放牧强度增加其频数 减少, 木地肤则相反, 也有物种频数在轻度放牧的
草地较小或为 0, 但可在重度放牧和不放牧的草地 生存(狗尾草、阿尔泰狗娃花)。

此外, 从植物对草食动物采食的响应模式来看, 草地植物多样性的差异主要由多年生杂类草和一二 年生草本两个功能群的部分物种引起, 即放牧条件 下, 二型叶棘豆、冷蒿、二裂委陵菜、陇牛儿苗、 画眉草等均未出现, 主要是因为这些物种本身并不 能很好地适应放牧干扰 (Milchunas et al., 1988; Gillson \& Hoffman, 2007), 在群落中地位较低, 分 布较少(孙世贤等, 2013), 且大都适口性较好, 极易 受到动物的采食, 在轻度放牧下就开始减少甚至灭 
绝消失(Zheng et al., 2011)。研究认为, 群落中优势 种占有大量资源, 直接或间接地控制着其他物种的 存活, 拥有绝对的统治地位, 对生态系统功能起主 导作用(Kraft et al., 2011; Mouillot et al., 2013); 对 稀有种而言, 其遭受干扰或破坏的过程中, 相应的 生物多样性和生态功能都异常脆弱, 因此, 判断生 物多样性高低的重要指标即群落中是否拥有大量的 稀有种(Lyons et al., 2005; Mouillot et al., 2013)。

\section{2 不同尺度上植物多样性对草食动物的响应}

放牧通过改变局部植物种的定植与灭绝速率, 动态调控植物群落的多样性, 当局部物种的灭绝速 率高于定植速率时, 引起局部物种多样性的降低 (Olff \& Ritchie, 1998); 也有研究认为, 放牧条件会 改变草地物种间的竞争强度, 致使一些在竞争上占 劣势的植物种因竞争而被排除, 导致群落中物种多 样性降低(卫智军等, 2013)。本研究的结果(图2)与之 较一致, 即群落尺度下, 物种多样性指数随放牧强 度增加而显著减少, 相比不放牧小区, 重度放牧与 适度放牧小区植物多样性指数分别下降 $10.92 \%$ 、 4.81\%; 但物种丰富度无显著性差异。这可能是因为 绵羊采食使草地出现较为可观的空余生态位, 增加 种库植物可植入的利用位点(Olff \& Ritchie, 1998), 间接地提高了草地植物的可分布范围, 加之短花针 茅荒漠草地本身结构简单, 物种数较少, 优势种和 耐牧物种比重较大(卫智军等, 2013), 同时丰富度指 数也只考虑了调查样方内的物种数量, 并没有体现 稀有种对物种丰富度的贡献量。但整体而言, 放牧 影响了群落中敏感物种的存活, 进而使群落内物种 多样性呈现随放牧强度增加而降低的格局(Gibson, 2009)。

而当研究尺度转化到群落内功能群时, 发现适 度放牧功能群多样性大于不放牧小区, 且显著大于 重度放牧小区。这一结果似乎与前文的论述相矛盾, 为了揭示其潜在原因, 本研究将尺度缩小并聚焦于 每个功能群内物种, 发现仅灌木半灌木的物种丰富 度产生变化, 不放牧处理和适度放牧处理显著大于 重度放牧 $(p<0.05)$; 物种多样性方面, 重度放牧草 地灌木半灌木和多年生杂类草物种多样性较不放牧 区分别显著下降了 $70.67 \% 、 22.97 \%$, 说明灌木半灌 木这一功能群内物种多样性对放牧干扰较敏感。这 是因为植物多样性是基于低组织尺度的数量特征来 获得高组织尺度研究范畴的运算方法, 表征的是低
组织尺度的植物多样性, 但却是高组织尺度的概念, 即通常研究的物种多样性体现的却是群落这一尺度 的概念。物种多样性与功能群多样性尽管均体现了 群落这一尺度的概念, 但却是基于群落内物种和功 能群的数量特征计算而来。尽管功能群尺度的数量 特征由物种计算而来, 但由此得出的植物多样性结 果却截然相反, 这在一定程度上说明一个或几个物 种的数量特征可能不会影响不同放牧强度植物功能 群多样性, 但却可能会对群落内物种、功能群内物 种多样性产生一系列级联反应, 导致灌木半灌木这 一功能群内物种多样性对家畜采食较敏感, 证明了 单个尺度仅能体现现存植物多样性一个研究维度的 特征。

\section{3 草食动物偏食性与荒漠草地植物多样性}

放牧对草地群落内物种的选择性强度是影响植 被组成的关键因素, 植物既是动物的直接食物来源, 也是形成动物采食生境的一个重要基础, 而植物多 样性在相当大程度上决定着动物食物的丰度和质 量。因此, 为了进一步探索荒漠草地植物多样性与 动物的潜在关系, 我们分析了不同放牧强度下绵羊 对各物种的喜食程度(图4), 发现偏食植物功能群为 一二年生草本和多年生杂类草, 这说明草食动物在 采食过程中表现出明显的选择性，几种或几类植物 成为采食食谱的主体构成成分, 而大多数植物在绵 羊日粮构成中比例较低(卫智军等, 2013)。研究认为 物种间的营养差别越大, 动物对它们的偏食性或选 择性也越大, 且绵羊对质地柔软、嫩叶丰富、籽实 丰富的植物有强偏食性, 如二型叶棘豆、乳白黄耆、 冷蒿、蒙古非、韭、细叶非等(图4)。然而, 动物的 食性选择及影响因素是极其复杂的, 也非一成不 变。本研究中, 绵羊对短花针茅、寸草、木地肤、 狭叶锦鸡儿的偏食性指数随放牧梯度增加减小, 而 无芒隐子草、阿尔泰狗娃花、栉叶高等却刚好相反, 这可能是因为当食物充足能满足其多种营养需求 时, 动物采食是以偏食物种为主且选择多样化的植 物组合来满足其营养平衡; 当食物相对紧张时, 动 物采食选择范围相对被动, 相应地以饱腹为目的, 侧面体现出动物采食并不仅仅局限于自身喜好或其 最大能量摄入量, 而是试图满足营养物质的需要量 而调节其采食选择(Simpson et al., 2004), 来实现其 最大适合度。也有研究认为, 当动物面临更复杂的 采食环境(如环境胁迫或竞争者增加), 动物采食后 
的反馈学习机制对其食性选择的作用将大大减弱 (Provenza, 1995; Villalba \& Provenza, 2000), 致使绵 羊的采食从更为积极地选择性采食转换为消极被动 地随机采食。此外, 我们对家畜的偏食性与草地的 多样性进行了相关分析, 发现动物偏食性与植物多 样性基本呈负相关关系, 这证明了在短花针茅 + 无 芒隐子草+碱非荒漠草地的物种较少的群落中, 绵 羊的采食行为会降低草地植物多样性 (Proulx \& Mazumder, 1998; Liu et al., 2015)。

综上所述, 本研究从既有放牧草地植物多样性 现状出发, 结合造成这一现状的草食动物的偏食性 这一视角来解释荒漠草地植物多样性对放牧的响应 机制, 结果表明: 1)荒漠草地植物对草食动物采食 呈现4种响应模式: 放牧“隐没种”、放牧“敏感种”、 放牧“无感种”、“绝对优势种”; 2)植物多样性受放牧 的影响, 可由物种尺度导致功能群尺度、群落尺度 甚至生态系统尺度的级联反应; 3)草食动物对植物 功能群偏食性的排序为一二年生草本 $>$ 多年生杂类 草>灌木半灌木>多年生禾草, 这一行为会降低荒漠 草地植物多样性。

基金项目 国家自然科学基金(31460126)、国家重 点基础研究发展计划(2014CB138805)、国家科技支 撑计划 (2012BAD13B00)和内蒙古自然科学基金 (2015MS0349)。

致谢 感谢中国农业科学院草原研究所孙世贤博士 在野外工作与论文写作过程中给予的帮助。

\section{参考文献}

Bai YF, Wu JG, Clark CM, Pan QM, Zhang LX, Chen SP, Wang QB, Han XG (2012). Grazing alters ecosystem functioning and C:N:P stoichiometry of grasslands along a regional precipitation gradient. Journal of Applied Ecology, 49, 1204-1215.

Chapin FS III, Zavaleta ES, Eviner VT, Naylor RL, Vitousek PM, Reynolds HL, Hooper DU, Lavorel S, Sala OE, Hobbie SE, Mack MC, Diaz S (2000). Consequences of changing biodiversity. Nature, 405, 234-242.

Chen DM, Zheng SX, Shan YM, Taube F, Bai YF (2013). Vertebrate herbivore-induced changes in plants and soils: Linkages to ecosystem functioning in a semi-arid steppe. Functional Ecology, 27, 273-281.

Deng RG (2012). Analysis on Soundscape Quality of Forest. $\mathrm{PhD}$ dissertation, Jiangxi Agricultural University, Nanchang. 63-84. (in Chinese) [邓荣根 (2012). 森林声 景观品质分析. 博士学位论文, 江西农业大学, 南昌. 63-84.]
Gibson DJ (2009). Grasses and Grassland Ecology. Oxford University Press, Oxford, UK.

Gillson L, Hoffman MT (2007). Rangeland ecology in a changing world. Science, 315, 53-54.

Hector A, Schmid B (1999). Plant diversity and productivity experiments in European grasslands. Science, 286, 1123-1127.

Hodgson J, Illius A (1996). The Ecology and Management of Grazing Systems. Oxford University Press, Oxford, UK.

Jared JB, Daniel L, Hernandez, Jae RP, Erika SZ (2015). Grazing maintains native plant diversity and promotes community stability in an annual grassland. Ecological Applications, 25, 1259-1270.

Kraft NJB, Comita LS, Chase JM, Sanders NJ, Swenson NG, Crist TO, Stegen JC, Vellend M, Boyle B, Anderson MJ, Cornell HV, Davies KF, Freestone AL, Inouye BD, Harrison SP, Myers JA (2011). Disentangling the drivers of $\beta$-diversity along latitudinal and elevational gradients. Science, 333, 1755-1758.

Liu J, Feng C, Wang D, Wang L, Wilsey BJ, Zhong Z (2015). Impacts of grazing by different large herbivores in grassland depend on plant species diversity. Journal of Applied Ecology, 52, 1053-1062.

Liu WT, Wei ZJ, Lü SJ, Sun SX, Dai JZ, Yan BL (2015). Above-ground biomass in Stipa breviflora desert grassland at different organizational scales. Chinese Journal of Applied \& Environmental Biology, 21, 912-918. (in Chinese with English abstract) [刘文亭, 卫智军, 吕世杰, 孙世 贤, 代景忠, 间宝龙 (2015). 短花针茅荒漠草原不同组 织尺度地上生物量. 应用与环境生物学报, 21 , 912-918.]

Louault F, Pillar VD, Aufrère J, Garnier E, Soussana JF (2005). Plant traits and functional types in response to reduced disturbance in a semi-natural grassland. Journal of Vegetation Science, 16, 151-160.

Lü SJ, Liu HM, Wu YL, Wei ZJ, Nie YQ (2014). Effects of grazing on spatial distribution relationships between constructive and dominant species in Stipa breviflora desert steppe. Chinese Journal of Applied Ecology, 25, 3469-3474. (in Chinese with English abstract) [吕世杰, 刘红梅, 吴艳玲, 卫智军, 聂雨芉 (2014). 放牧对短花 针茅荒漠草原建群种与优势种空间分布关系的影响. 应用生态学报, 25, 3469-3474.]

Lyons KG, Brigham CA, Traut BH, Schwartz MW (2005). Rare species and ecosystem functioning. Conservation Biology, 19, 1019-1024.

Ma KP (1993). Try theory the concept of biodiversity. Biodiversity Science, 1, 20-22. (in Chinese with English abstract) [马克平 (1993). 试论生物多样性的概念. 生物 多样性, 1, 20-22.]

Ma YS, Zhang ST (2009). Responses of plant from individual to community level to grazing: A review. Chinese Journal

www.plant-ecology.com 
of Ecology, 28, 113-121. (in Chinese with English abstract) [马银山, 张世挺 (2009). 植物从个体到群落水 平对放牧的响应. 生态学杂志, 28, 113-121.]

Milchunas DG, Sala OE, Lauenroth WK (1988). A generalized model of the effects of grazing by large herbivores on grassland community structure. American Naturalist, 132, 87-106.

Mouillot D, Bellwood DR, Baraloto C, Chave J, Galzin R, Harmelin-Vivien M, Kulbicki M, Lavergne S, Lavorel S, Mouquet N (2013). Rare species support vulnerable functions in high-diversity ecosystems. PLoS Biology, 11, $1-11$.

Olff H, Ritchie ME (1998). Effects of herbivores on grassland plant diversity. Trends in Ecology \& Evolution, 13, 261-265.

Proulx M, Mazumder A (1998). Reversal of grazing impact on plant species richness in nutrient-poor vs. nutrient-rich ecosystems. Ecology, 79, 2581-2592.

Provenza FD (1995). Postingestive feedback as an elementary determinant of food preference and intake in ruminants. Journal of Range Management, 48, 2-17.

Rossignol N, Bonis A, Bouzille JB (2006). Consequence of grazing pattern and vegetation structure on the spatial variations of $\mathrm{N}$ mineralization in a wet grassland. Applied Soil Ecology, 31, 62-70.

Simpson SJ, Sibly RM, Lee KP, Behmer ST, Raubenheimer D (2004). Optimal foraging when regulating intake of multiple nutrients. Animal Behaviour, 68, 1299-1311.

Stephens DW, Krebs JR (1986). Foraging Theory. Princeton University Press, Princeton, USA.

Storkey J, Macdonald AJ, Poulton PR, Scott T, Köhler IH, Schnyder H, Goulding KWT, Crawley MJ (2015). Grassland biodiversity bounces back from long-term nitrogen addition. Nature, 528, 401-404.

Sun RY, Li B, Zhuge Y, Shang YC (1993). General Ecology. Higher Education Press, Beijing. (in Chinese) [孙儒泳, 李 博, 诸葛阳, 尚玉昌 (1993). 普通生态学. 高等教育出 版社, 北京.]

Sun SX, Wei ZJ, Lü SJ, Lu ZH, Chen LB, Li XZ, Wu YL, Li JR (2013). Characteristics of plant community and its functional groups in desert grassland under effects of seasonal regulation of grazing intensity. Chinese Journal of Ecology, 32, 2703-2710. (in Chinese with English abstract) [孙世贤, 卫智军, 吕世杰, 卢志宏, 陈立波, 李 夏子, 吴艳玲, 李建茹 (2013). 放牧强度季节调控下荒 漠草原植物群落与功能群特征. 生态学杂志, 32, 27032710.]

Tilman D, Isbell F (2015). Biodiversity: Recovery as nitrogen declines. Nature, 528, 336-337.

Villalba JJ, Provenza FD (2000). Discriminating among novel foods: Effects of energy provision on preferences of lambs for poor-quality foods. Applied Animal Behaviour Science, 66, 87-106.

Wan HW, Bai YF, Schönbach P, Gierus M, Taube F (2011). Effects of grazing management system on plant community structure and functioning in a semiarid steppe: Scaling from species to community. The Plant Soil, 340, 215-226.

Wang L (2010). Behavioral Adaptive Mechanisms of Large Herbivore Foraging in Response to Diversity and Spatial Pattern of Plants. PhD dissertation, Northeast Normal University, Changchun. 39-49. (in Chinese with English abstract) [王岭 (2010). 大型草食动物采食对植物多样 性与空间格局的响应及行为适应机制. 博士学位论文, 东北师范大学, 长春. 39-49.]

Wang L, Wang D, Bai Y, Huang Y, Fan M, Liu J, Li Y (2010). Spatially complex neighboring relationships among grassland plant species as an effective mechanism of defense against herbivory. Oecologia, 164, 193-200.

Wei ZJ, Han GD, Zhang G, Li DX (2013). Research of Chinese Desert Grassland Ecosystem. Science Press, Beijing. (in Chinese) [卫智军, 韩国栋, 赵钢, 李德新 (2013). 中国 荒漠草原生态系统研究. 科学出版社, 北京.]

Xu YF, Yixicuomu, Fu JJ, Chen H, Miao YJ, Chen J, Hu TM, Zhong JG (2012). Response of plant diversity and soil nutrient to grazing intensity in Kobresia pygmaea Meadow of Qinghai-Tibet Plateau. Acta Agrestia Sinica, 20, 10261032. (in Chinese with English abstract) [许岳飞, 益西措 姆, 付娟娟, 陈浩, 苗彦军, 陈俊, 呼天明, 镡建国 (2012). 青藏高原高山嵩草草甸植物多样性和土壤养分 对放牧的响应机制. 草地学报, 20, 1026-1032.]

Yang J, Chu PF, Chen DM, Wang MJ, Bai YF (2014). Mechanisms underlying the impacts of grazing on plant $\alpha, \beta$ and $\gamma$ diversity in a typical steppe of the Inner Mongolia grassland. Chinese Journal of Plant Ecology, 38, 188-200. (in Chinese with English abstract) [杨婧, 褚鹏飞, 陈迪马, 王明玖, 白永飞 (2014). 放牧对内蒙古典型草原 $\alpha 、 \beta$ 和 $\gamma$ 多样性的影响机制. 植物生态学报, 38, 188-200.]

Zheng SX, Lan ZC, Li WH, Shao RX, Shan YM, Wan HW, Taube F, Bai YF (2011). Differential responses of plant functional trait to grazing between two contrasting dominant $\mathrm{C}_{3}$ and $\mathrm{C}_{4}$ species in a typical steppe of Inner Mongolia, China. Plant and Soil, 340, 141-155.

责任编委: 王德利 责任编辑: 李 敏 


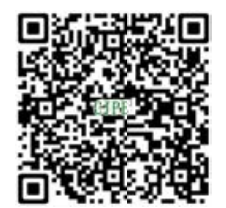

植物生态学报官网

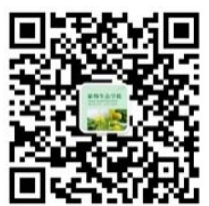

微信订阅号

期刊及学科

相关信息发布

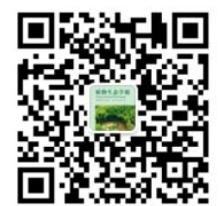

微信服务号

稿件状态查询

全文检索浏览 